\title{
Fuzzified Coulomb's and Franklin's laws behaved optimization for economic dispatch in multi-area multi-fuel power system
}

\author{
V. Ponnuvel Sakthivel ${ }^{1} \cdot$ P. Duraisamy Sathya ${ }^{2}$
}

Received: 29 May 2020 / Accepted: 21 December 2020 / Published online: 11 January 2021

(c) The Author(s) 2021 OPEN

\begin{abstract}
Multi-Area Multi-Fuel Economic Dispatch (MAMFED) aims to allocate the best generation schedule in each area and to offer the best power transfers between different areas by minimizing the objective functions among the available fuel alternatives for each unit while satisfying various constraints in power systems. In this paper, Fuzzified Coulomb's and Franklin's Laws Behaved Optimization (FCFLBO) approach is proposed to solve the MAMFED problem. Coulomb's and Franklin's Laws Behaved Optimization (CFLBO) approach is developed from Coulomb's and Franklin's theories, which encompass fascination/aversion, ionization, and contact stages. The suggested approach considers the line losses, valve point loading impacts, multi-fuel alternatives, and tie-line limits of the power system. Because of the contradicting nature of fuel cost and pollutant emission objectives, weighted sum approach and price penalty factor are used to transfer the bi-objective function into single objective function. Furthermore, a fuzzy decision strategy is introduced to find one of the Pareto optimal fronts as the best comprised solution. The feasibility of the FCFLBO algorithm is tested on a three-area test system for both the single-area multi-fuel economic dispatch and MAMFED problems. The results of FCFLBO algorithm are compared with those of the krill herd algorithm, exchange market algorithm and other heuristic approaches surfaced in the literature. To show the effectiveness of FCFLBO algorithm, multi-objective performance indicators such as generational distance, spacing metric and ratio of non-dominated individuals are evaluated. The results divulge that the FCFLBO is a promising approach to solve the MAMFED problem as it furnishes better compromised solution in comparison with the other heuristic approaches.
\end{abstract}

Keywords Fuzzy set theory · Heuristic optimization · Multi-area economic dispatch · Pareto-optimal front · Tie-line constraint

\begin{tabular}{|c|c|c|c|}
\hline Abbreviations & & $a_{0}$ and $r_{0}$ & Initial values for positive and negative \\
\hline$a_{i}, b_{i}, c_{i}$ & Cost coefficients of generator $i$ for single & & charges respectively \\
\hline & area power system & $B_{i j}$ & Line loss coefficients \\
\hline$a_{i j}, b_{i j}, c_{i j}$ & $\begin{array}{l}\text { Cost coefficients of generator } i \text { in area } j \\
\text { for multi-area power system }\end{array}$ & $d_{i}, e_{i}$ & $\begin{array}{l}\text { Cost coefficients of the VPL effect of } \\
\text { generator } i \text { in single area power system }\end{array}$ \\
\hline$a_{i j k}$ & $\begin{array}{l}\text { Cost coefficients of generator } i \text { in area } \\
j \text { for fuel type } k \text { in multi-area multi-fuel } \\
\text { power system }\end{array}$ & $e_{i j j} f_{i j}$ & $\begin{array}{l}\text { Cost coefficients of the VPL effect of } \\
\text { generator } i \text { in area } j \text { for multi-area power } \\
\text { system }\end{array}$ \\
\hline$a_{\max }$ and $r_{\max }$ & $\begin{array}{l}\text { Maximum number of positive and nega- } \\
\text { tive charges respectively }\end{array}$ & $E_{i}$ & $\begin{array}{l}\text { Emission of the generator } i \text { in single area } \\
\text { power system }\end{array}$ \\
\hline
\end{tabular}

$\triangle$ V.Ponnuvel Sakthivel, vp.sakthivel@yahoo.com; P. Duraisamy Sathya, pd.sathya@yahoo.in | ${ }^{1}$ Department of Electrical and Electronics Engineering, Government College of Engineering, Dharmapuri 636704, Tamilnadu, India. ${ }^{2}$ Department of Electronics and Communication Engineering, Faculty of Engineering and Technology, Annamalai University, Chidambaram 608002, Tamilnadu, India. 


\begin{tabular}{|c|c|}
\hline$F_{i}$ & $\begin{array}{l}\text { Fuel cost of the generator } i \text { in single area } \\
\text { power system }\end{array}$ \\
\hline$F_{i j}\left(P_{i j}\right)$ & $\begin{array}{l}\text { Fuel cost of the generator } i \text { in area } j \text { for } \\
\text { multi-area power system }\end{array}$ \\
\hline$F_{j}^{\max }$ and $F_{j}^{\min }$ & $\begin{array}{l}\text { Maximum and minimum values of } j \text { th } \\
\text { objective function respectively }\end{array}$ \\
\hline$F_{\mathrm{bcs}}$ and $E_{\mathrm{bcs}}$ & Cost and emission obtained by EED \\
\hline$F_{\min }$ and $E_{\max }$ & $\begin{array}{l}\text { Cost and emission obtained by ED mini- } \\
\text { mization respectively }\end{array}$ \\
\hline$F_{\max }$ and $E_{\min }$ & $\begin{array}{l}\text { Cost and emission obtained by } \mathrm{EmD} \\
\text { minimization respectively }\end{array}$ \\
\hline$h$ & Price penalty factor in $\$ / \mathrm{h}$ \\
\hline IP & Improvement percentage \\
\hline$k$ & Number of fuel alternatives \\
\hline$m$ & Population size of each object \\
\hline$M_{i}$ & $\begin{array}{l}\text { Number of participated generators in } \\
\text { area } i\end{array}$ \\
\hline M & Number of non-dominated solutions \\
\hline$n$ & Maximum number of objects \\
\hline$n g$ & Total number of generating units \\
\hline$P_{D}$ & Power demand \\
\hline$P_{i}$ & $\begin{array}{l}\text { Real power generation of generator } i \text { in } \\
\text { single area power system }\end{array}$ \\
\hline$P_{i j}$ & $\begin{array}{l}\text { Real power generation of generator } i \text { in } \\
\text { area } j \text { for multi-area power system }\end{array}$ \\
\hline$P_{i, \min { }^{\prime}} P_{i, \max }$ & $\begin{array}{l}\text { Minimum and maximum generation of } \\
\text { unit } i \text { in single area power system }\end{array}$ \\
\hline$P_{i j, \min n^{\prime}} P_{i j, \max }$ & $\begin{array}{l}\text { Minimum and maximum generation } j \text { in } \\
\text { area } i \text { for multi-area power system }\end{array}$ \\
\hline$p_{i}$ & Ionization probabilistic constant \\
\hline$P_{c}$ & Contact phase probabilistic constant \\
\hline$T_{i z}$ & $\begin{array}{l}\text { Tie line power stream from area } i \text { to area } \\
z\end{array}$ \\
\hline$T_{i z, \max }$ & $\begin{array}{l}\text { Maximum tie line power stream from } \\
\text { area } i \text { to area } z\end{array}$ \\
\hline$-T_{i z, \max }$ & $\begin{array}{l}\text { Maximum tie line power stream from } \\
\text { area } z \text { to area } i\end{array}$ \\
\hline$w$ & Weight or compromise factor \\
\hline$x_{i j}$ & $\begin{array}{l}j^{\text {th }} \text { elementary charge of the } i^{\text {th }} \text { point } \\
\text { charge }\end{array}$ \\
\hline$x_{j}^{\min }$ and $x_{j}^{\max }$ & $\begin{array}{l}\text { Minimum and maximum limits of } \\
\text { variable } j\end{array}$ \\
\hline$\theta_{j}^{\text {new }}$ & $\begin{array}{l}\text { Current electric angle of } j \text { th elementary } \\
\text { charge in radians }\end{array}$ \\
\hline$\theta_{j}^{\text {old }}$ & $\begin{array}{l}\text { Previous electric angle of } j \text { th elemen- } \\
\text { tary charge in radians }\end{array}$ \\
\hline$a_{i}, \beta_{i}, \gamma_{i}$ & Emission coefficients of generator $i$ \\
\hline
\end{tabular}

\section{Introduction}

The goal of MAMFED problem is to decide the power delivered by every generator in various areas and the power stream between the areas in order to lessen the total production cost and pollutants outflows of the interconnected power system considering multi-fuel alternatives of each generating unit. The total load power is appropriated equally among various areas to decrease the fuel cost as well as the pollutant emissions. The power scheduling between the zones must not disregard the power balance, generator and tie-line limits. Thus, the MAMFEED problem is addressed as the large-scale highly non-linear multi-objective optimization problem. In recent years, the MAMFED problem has received considerable attention.

Over the years, various classical multi-area power generation scheduling methods have been proposed. Shoults, et al. [1] applied an efficient approach for unit commitment and Economic Dispatch (ED) problems with area import/export constraints. The proposed approach has been tested on the Texas utilities and Texas municipal power pool systems. Quintana et al. [2] suggested the use of the Dantzig-Wolfe decomposition principle regarding the revised simplex method and a fast-decoupled power flow algorithm for constrained Multi-Area ED (MAED) of power systems. Ouyang and Shahidehpour [3] developed a model of large-scale multi-area power generation system and used a rule-based heuristic strategy to improve the generation schedule for every zone. Wang and Shahidehpour [4] suggested a decomposition approach which upgrades the scheduling process and accelerates the execution of a large-scale multi-area generation system in a real-time application. Streiffert [5] expressed the MAED problem as a capacitated nonlinear network flow problem and solved it through an Incremental Network Flow Programming (INFP) approach. The results showed that the INFP approach was fast, robust, and extendable to large-scale systems. Yalcinoz and Short [6] presented an Improved Hopfield Neural Network (IHNN) to solve MAED problems with transmission capacity constraints and showed that the IHNN achieved efficient and accurate solutions for two-area power systems. Thang [7] proposed a Hopfield Lagrange network for solving economic emission dispatch (EED) problem with multiple fuel alternatives (MFAs). Furthermore, the best compromise from the set of obtained solutions was found and compared with from LamdaIteration method. These techniques are probably to come upon severe difficulty due to their heavy imposition of diverse constraints such as continuity, convexity and differentiability on the objective functions, and 
high sensitivity to the initial values of involved optimized variables. Furthermore, their performances are progressively worsened by the dimension of the problem. Thus, these strategies do not offer reasonable possibilities for dealing with the MAED problem when prohibited operating zones (POZs), valve point loading (VPL), and MFAs are considered. To adapt to these challenges, numerous meta-heuristic methodologies have been used to take care of enhancement issues with complicated objectives [8]. The results obtained by evolutionary approaches for complex optimizing problems are good, which means these approaches offer a reasonable possibility for solving MAED problems.

In recent years, swarm intelligence algorithms have been broadly used to overcome the computational unpredictability issues in the MAED problem. Jayabarathi et al. [9] proposed a proficient technique for MAED problems using an Evolutionary Programming (EP) approach. The performance of the various evolutionary algorithms, for example the Real-Coded Genetic Algorithm (RCGA), Particle Swarm Optimization (PSO), Differential Evolution (DE), and the Covariance Matrix Adapted Evolution Strategy (CMAES), on MAED problems with Karush-Kuhn-Tucker optimality conditions was examined [10]. The simulation results revealed that the CMAES algorithm offers the best results and the Nelder-Mead simplex method offers an optimal solution in the shortest time among the different algorithms considered. The performance of different DE strategies enhanced with time-varying mutation was investigated and analyzed to solve the reserve-constrained MAED problem [11]. The time varying DE approach was found to be capable of determining better solutions than PSO with time-varying acceleration coefficients. Also, the DE variant approaches were able to find the global best solutions for large-scale systems, while basic DE suffered from premature convergence.

Somasundaram and Jothi Swaroopan [12] introduced another computationally efficient fuzzified PSO algorithm to solve the security-constrained MAED problem of an interconnected power system. The inertia weight of the standard PSO was made adaptive by using fuzzy logic strategies to improve the convergence speed and avoid premature convergence. Basu proposed artificial bee colony $(A B C)$ optimization [13] to solve a MAED problem with tie-line constraints, transmission losses, multiple fuels, and valve point effects. Evolutionary approaches such as $D E, E P$, and RCGA were applied to analyze the efficiency of the $A B C$ approach. The simulation results showed that the $A B C$ approach converged to a better solution than the approaches with which it was compared. Teaching learning-based optimization (TLBO) [14] has been applied to solve the MAED issue. The approach was tested on 3 different systems and the results revealed that the presented approach had the ability to provide better solutions and exhibited greater robustness than DE, EP, and RCGA. The different MAED models were solved by employing Fast Convergence Evolutionary Programming (FCEP) [15]. FCEP used Gaussian and Cauchy mutations to improve the convergence speed and solution quality. Nguyen et al. [16] developed the Hybrid Cuckoo Search Algorithm (HCSA) to solve the MAED problem. The HCSA consolidates the cuckoo search algorithm and TLBO to improve the recital of cuckoo eggs. Ghasemi et al. [17] presented a hybrid DEPSO technique to address the MAED, reserve-constrained MAELD, and reserve-constrained multi-area ecological/ economic dispatch problems.

Sum Differential Evolution with Particle Swarm Optimizer (SDEPSO) was used along with the local (Pbest) optimal value in a DE crossover operator. The simulation results showed that the hybrid algorithm achieved a balance between global search ability and provision of better convergence and solution quality. Zhang et al. [18] introduced an Improved Grasshopper Optimization Algorithm (IGOA) to deal with the MAED problem. A chaos mechanism was adopted in the IGOA to handle the premature convergence of the grasshopper optimization algorithm. The simulation results demonstrated that the IGOA could converge better than the other approaches. Modiri-Delshad et al. [19] presented Backtracking Search Algorithm (BSA) for solving ED problems with considering VPL effects, POZs, and MFAs. Different variants of Krill Herd Algorithm (KHA) to small, medium and largescale power systems for solving the ELD problems were applied by Mandal et al. [20]. Ghorbani and Babaei presented Exchange Market Algorithm (EMA) for solving the ELD problems. The EMA was employed by 2 searchers and absorbent operators which resulted in generation and organization of random numbers in the best way [21]. The MAED problem was solved by the hybridization of JAYA and TLBO approaches to simultaneously solve both non-smooth and non-convex characteristics [22]. A Parallel MAED (PMAED) was developed to maintain the independency and transfer the required power in each area [23]. The PMAED was solved with cost characteristics of different complexities. A Newton Method based Distributed Algorithm (NMDA) was employed to solve Single-Area Economic Dispatch (SAED) and MAED problems [24]. The virtual SAED formulation, backtracking line search algorithm, and average consensus theory were embedded in the NMDA approach. In [25] an improved fireworks algorithm (IFA) was used to solve the multiarea ELD problem considering the valve point loading (VPL) effects. The tie-line limit between different areas, generation limits, ramp rate limits, transmission losses, prohibited operating zone (POZ) and spinning reserve as the problem constraints. In the IFA, cross-generation 
mutation mechanisms were employed to solve the multiconstrained the multi-area ELD problem. A novel swarm intelligence approach using salp swarm algorithm for the solution of multi-area generation scheduling with wind integration was presented [26]. These improved and hybrid forms of heuristic approaches involve complicated computation owing to the use of many control parameters. An efficient algorithm to solve MAED problems to ascertain the optimum dispatch solutions must be developed and proposed.

A new swarm intelligence approach, Squirrel Search Algorithm (SSA) was developed to solve the ELD of thermal units by addressing VPL and MFO for single area power system [27]. Recently, Sakthivel et al. [28] applied SSA and Coulomb's and Franklin's Laws Based Optimizer (CFLBO) [29] to solve the economic environmental dispatch of single area power system. In these research works; the multiple fuel options and multi-area power systems were not considered for the solution of economic environmental dispatch problem.

In 2018, a new physics-motivated approach, CFLBO was introduced by Ghasemi et al. [30]. This approach is a populace-based methodology propelled by Coulomb's and Franklin's speculations. The powers of fascination and repugnance between the point charges, ionization and contact stages are contemplated. CFLBO is beneficially used in ED issues with different complexities and is superior to other approaches surfaced in the literature. The special features of the CFLBO algorithm are as follows:

i. The attraction/repulsion stage is used to increase the learning ability of the population and to enhance the global search ability of the algorithm.

ii. The probabilistic ionization and contact stages are used to increase the population diversity and to avoid premature convergence of the algorithm.

Due to the above characteristics, the CFLBO approach is able to provide solutions of better quality than the existing heuristic approaches.

The main contributions of this paper are briefly summarized as follows:

- This paper aims to first introduce the FCFLBO algorithm to solve the MAMFED problem. The proposed algo- rithm involves the CFLBO algorithm, weighted sum approach and fuzzy decision strategy.

- The FCFLBO algorithm successfully solves the Single Area Multi-Fuel ED (SAMFED) such that the fuel costs and pollutant emissions are simultaneously minimized while fulfilling the power balance and generation limits. Furthermore, the suggested algorithm is successfully applied to solve the MFMAED such that the total fuel cost is minimized while fulfilling the system and area constraints.

- The suggested FCFLBO, KHA and EMA are applied on a three-area 10-units system in order to demonstrate its effectiveness in solving the SAMFED and MAMFED problems. The obtained results are compared with different state-of-theart heuristic approaches surfaced in the literature.

The structure of this paper is as follows. Section 2 presents the details of the SAMFED and MAMFED models. The recommended FCFLBO is presented in Sect. 3. The implementation of the FCFLBO approach for theMAMFED problem is discussed in Sect. 4 . In Sect. 5, the numerical results are discussed and the adequacy of the proposed FCFLBO approach is additionally demonstrated. Finally, Sect. 6 concludes the paper.

\section{Problem formulation of multi-fuel economic dispatch}

\subsection{SAMFED}

\subsubsection{Fuel cost objective function}

The goal of the ED problem is to limit the all-out expense of thermal units as follows:

Minimize $F=\sum_{i=1}^{n g} F_{i}\left(P_{i}\right)$

In the ED problem, the fuel cost of each generating unit is communicated as a quadratic function of its capacity yield. As the generating units utilize different fuel choices to create power and the valve point impacts considered, the fuel cost function is expressed as

$F_{i}\left(P_{i}\right)=\left\{\begin{array}{lll}\text { Fuel type } 1 ; & a_{i 1}+b_{i 1} P_{i}+c_{i 1} P_{i}^{2}+\left|e_{i 1} \times \sin \left(f_{i 1} \times\left(P_{i, \min }-P_{i}\right)\right)\right| ; & P_{i, \min } \leq P_{i} \leq P_{i 1} \\ \text { Fuel type } 2 ; & a_{i 2}+b_{i 2} P_{i}+c_{i 2} P_{i}^{2}+\left|e_{i 2} \times \sin \left(f_{i 2} \times\left(P_{i, \min }-P_{i}\right)\right)\right| ; & P_{i 1}<P_{i} \leq P_{i 2} \\ \cdots & & \\ \text { Fuel type } k ; & a_{i k}+b_{i k} P_{i}+c_{i k} P_{i}^{2}+\left|e_{i k} \times \sin \left(f_{i k} \times\left(P_{i, \min }-P_{i}\right)\right)\right| ; & P_{i k-1}<P_{i} \leq P_{i, \max }\end{array}\right.$ 


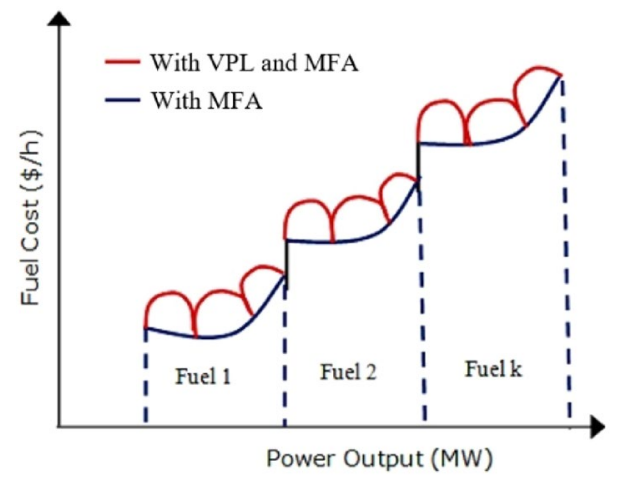

Fig. 1 Multi-fuel cost curve

The multi-fuel cost curve with and without VPL impacts is shown in Fig. 1.

\subsubsection{Emission objective function}

The objective of the emission dispatch (EmD) problem is to lessen the hazardous emissions as follows:

Minimize $E=\sum_{i=1}^{n g} E_{i}\left(P_{i}\right)$

The emission objective function is truly like the fuel cost function while it deals with all discharge types discharged by generation units. The scientific model for emanation function with MFA is introduced as

$E_{i}\left(P_{i}\right)=\left\{\begin{array}{lll}\text { Fuel type } 1 ; & \alpha_{i 1}+\beta_{i 1} P_{i}+\gamma_{i 1} P_{i}^{2} ; & P_{i, \text { min }} \leq P_{i} \leq P_{i 1} \\ \text { Fuel type } 2 ; & \alpha_{i 2}+\beta_{i 2} P_{i}+\gamma_{i 2} P_{i}^{2} ; & P_{i 1}<P_{i} \leq P_{i 2} \\ \cdots & & \\ \text { Fuel type } k ; & \alpha_{i k}+\beta_{i k} P_{i}+\gamma_{i k} P_{i}^{2} ; & P_{i, k-1}<P_{i} \leq P_{i, \max }\end{array}\right.$
Minimize $F_{E E D}=w \times F+h \times(1-w) \times E$

The above condition becomes ED target function when $\mathrm{w}=1$ and becomes EmD target work when $w=0 . w$ is a primary function of rand $[0,1]$ which bargains the fuel cost and emanation objectives.

\subsubsection{Power balance constraint}

The total power generated from a set of committed units must fulfil the total load demand.

$\sum_{i=1}^{n g} P_{i}=P_{D}$

\subsubsection{Generator capacity limits}

The real output power of thermal units ought to be in their range between minimum and maximum limits:

$P_{i, \min } \leq P_{i} \leq P_{i, \max }$

\subsection{MAMFED}

\subsubsection{Fuel cost objective function}

The aim of the MAMFED problem is to find the amount of power that can be efficiently generated in one area and transferred to another area, and to determine the economic fuel choice for each unit. Since generators are provided with multi-fuel sources, every generator ought to be defined with a few piecewise quadratic capacities superimposed by sine terms mirroring the impact of changes in the type of fuel and the generator must determine the most conservative fuel to consume. The MAED problem with VPL and MFA impacts [13] can be modeled as

$F_{i j}\left(P_{i j}\right)=\left\{\begin{array}{lll}\text { Fuel type } 1 ; & a_{i j 1}+b_{i j 1} P_{i j}+c_{i j 1} P_{i j}^{2}+\left|e_{i j 1} \times \sin \left(f_{i j 1} \times\left(P_{i j, m i n}-P_{i j}\right)\right)\right| ; & P_{i j, \min } \leq P_{i j} \leq P_{i j 1} \\ \text { Fuel type } 2 ; & a_{i j 2}+b_{i j 2} P_{i j}+c_{i j 2} P_{i j}^{2}+\left|e_{i j 2} \times \sin \left(f_{i j 2} \times\left(P_{i j, m i n}-P_{i j}\right)\right)\right| ; & P_{i j 1}<P_{i j} \leq P_{i j 2} \\ \ldots & \\ \text { Fuel type } k ; & a_{i j k}+b_{i j k} P_{i j}+c_{i j k} P_{i j}^{2}+\left|e_{i j k} \times \sin \left(f_{i j k} \times\left(P_{i j, \min }-P_{i j}\right)\right)\right| ; & P_{i j k-1}<P_{i j} \leq P_{i j, \max }\end{array}\right.$

\subsubsection{Economic and emission dispatch (EED) function}

The EED problem can be figured as bi-target work in which fuel cost and discharge as equalling objectives. This bi-target function can be moved to a solitary target function as follows:
The objective function for the MAMFED problem is defined as:

MinimizeF $=\sum_{j=1}^{M i} \sum_{i=1}^{n_{g}} F_{i j}\left(P_{i j}\right)$ 


\subsubsection{Power balance constraint}

The total power generated by a set of accessible units must satisfy the total load demand, tie-line power flow, and transmission losses [8] and is given by.

$\sum_{j=1}^{M_{i}} P_{i j}=P_{D i}+P_{L i}+\sum_{z, z \neq i} T_{i z} \quad i \in n_{g}, j \in M_{i}$

where $P_{D i}$ is the load demand in area $j ; P_{L i}$ represents the transmission line losses in area $\mathrm{i} ; \mathrm{T}_{\mathrm{iz}}$ is the tie-line power flow from area i to area $z$.

The transmission loss $P_{L j}$ of area $j$ can be defined by utilizing B-coefficients as

$P_{L i}=\sum_{l=1}^{M_{i}} \sum_{j=1}^{M_{i}} P_{i j} B_{i j} P_{i l}+\sum_{j=1}^{M_{i}} B_{0 i j} P_{i j}+B_{00 i}$

\subsubsection{Generator capacity limits.}

The real output power of the thermal units should be in the range between minimum and maximum limits [9]:

$P_{i j, \min } \leq P_{i j} \leq P_{i j, \max }$

\subsubsection{Tie-line limit.}

Because of the security basis, power shifted between various lines must not surpass their cutoff points [11]. The power transfer requirement between two unique regions is characterized by

$-T_{i z, \max } \leq T_{i z} \leq T_{i z, \max }$

\section{Brief overview of the FCFLBO Approach}

\subsection{CFLBO algorithm}

CFLBO is a heuristic algorithm, developed by Ghasemi et al. [30]. This methodology impersonates Coulomb's and Franklin's speculations. The accompanying ideas are related with CFLBO approach.

Coulomb's Law The association between two distinctive point charges is constrained by the extent of electrostatic intensity of fascination (or) repugnance.
Franklin's Law Every object involves equivalent positive and negative charges.

The CFLBO approach utilizes different objects of point charges $(X)$ which turn around different regions in a researched space to locate the global arrangement. The underlying items are framed by various gatherings of point charges which are self-assertively produced in the pursuit space. Each point charge includes $D$ quantized charges and each point charge is contrasted with a solution of the issue. The paradigm of CFLBO is a monotonous procedure, which contains initiation, fascination/aversion, ionization and contact stages.

\subsubsection{Initiation stage}

Let an object is generated by a populace of $m$ charges with variables $D$. The objects, populaces and each individual are indicated as follows [29]:

$O=\left[O_{1}, O_{2}, \ldots O_{n}\right]$

$X=\left[X_{1}, X_{2}, \ldots X_{q}\right]$

$X_{i j}=\left[x_{i 1}, x_{i 2}, \ldots x_{i D}\right]$

The underlying populaces of point charges are created as [29]:

$x_{i j}=U\left(x_{j}^{\min }, x_{j}^{\max }\right) \quad$ for $i=1,2, \ldots \mathrm{m}$ and $\mathrm{j}=1,2, . \mathrm{D}$

where $U$ is a vector of consistently disseminated arbitrary numbers from $x_{j}^{\min }$ to $x_{j}^{\max }$.

At that point, the underlying populace is organized and scattered into a couple of objects $\left(\mathrm{O}_{1} \ldots \ldots . . \mathrm{O}_{n}\right)$.

\subsubsection{Fascination/Aversion stage}

The migration of point charge is affected by fascination and aversion powers following up on them. The net force following upon a point charge $\left(X_{i}\right)$ is equivalent to its worth $\left(F_{i}\right)$. The CFLBO approach is utilized to restrict the net power following upon them. The point charges of each object are refreshed as [29]: 


$$
\begin{aligned}
x_{j}^{\text {new }}= & x_{i j}^{\text {old }}+\left|\cos \theta_{j}^{\text {new }}\right|^{2} \times\left(x_{j}^{\text {best }}-x_{j}^{\text {Worst }}\right) \\
& +\left|\sin \theta_{j}^{\text {new }}\right|^{2} \times\left(\operatorname{mean}\left(\sum_{n=1}^{a_{\max }} x_{j n}\right)-\operatorname{mean}\left(\sum_{n=1}^{r_{\max }} x_{j n}\right)\right)
\end{aligned}
$$

According to this equation, if the objective value of the new location $f\left(x_{j}^{\text {new }}\right)$ is preferable or less over that of the old positionf $\left(x_{i j}^{\text {old }}\right), x_{j}^{\text {new }}$ is set as the new location of $x_{j}$; or else, $x_{j}$ sustains its past position $x_{i j}^{\text {old }}$. The negative point charges with respect to $x_{j}$, which repulse $x_{j}$, seem with negative sign in the Eq. 15 to rise the separation between the charges.where, $\theta_{j}^{\text {initial }}=U(0,2 \pi)$

$$
\theta_{j}^{\text {new }}=\theta_{j}^{\text {old }}+U\left(0, \frac{3}{2} \pi\right)
$$

The $a_{\max } \mathrm{a}_{\max }$ and $r_{\max } r_{\max }$ are defined as:

$a_{\max }=a_{0} \times(1+\cos \theta)$

$r_{\max }=r_{0} \times(1-\cos \theta)$

\subsubsection{Probabilistic ionization stage}

Because of the impact of probabilistic ionization energy, there is a chance of removal of the position of the rudimentary charge $x_{j}$. It can be expressed by the accompanying condition [29].

$x_{j}^{\text {new }}=x_{j}^{\text {Best }}+x_{j}^{\text {Worst }}-x_{j}^{\text {old }}$ if $\operatorname{rand}(i) \leq p_{i}$

The parameter ' $j$ ' is selected as follows.

$j=\operatorname{round}($ unifrnd $(1, D))$

where rand (i)(i) is the ith point charge of a randomly generated number between zero and one.

If $P_{i}$ for $j$ th member $\left(x_{j}\right)$ is higher than $\operatorname{rand}(i)$, the variable $x_{j, k}$ is randomly chosen by Eq. 19 and the $k$ th control variable is updated using Eq. 18.

\subsubsection{Probabilistic contact stage}

In the event that the objects are engaging with one another, at that point each object elapses its best and worst charges for its neighborhood. The contact stage is communicated as [29]:

If rand $_{c} \leq p_{c}$, then

$x_{j}^{\text {Best }_{O b j 1}}=x_{j}^{\text {Best }_{O b j n}}, \ldots x_{j}^{\text {Best }_{O b j n}}=x_{j}^{\text {Best }_{O b j n-1}}$ $x_{j}^{\text {Worst }_{O b j 1}}=x_{j}^{\text {Worst }_{O b j n}}, \ldots x_{j}^{\text {Worst }_{O b j n}}=x_{j}^{\text {Worst }_{O b j n-1}}$

where rand $_{c}$ is a randomly generated number between zero and one.

If the factor $P_{c^{\prime}}$ is higher than rand, then each object elapses its greatest and lowest cost values of charges for its neighborhood using Eq. (20).

\section{Fuzzy decision strategy}

Fuzzy set hypothesis is used to proficiently determine the best compromised solution (BCS) from a Pareto optimal fronts (POF) set. Generally, a membership function (MF) for every objective function is expressed by the expertness and instinctive information. A basic straight MF is used for every objective function. It can be expressed as [29]:

$\mu\left(F_{j}\right)= \begin{cases}1 & \text { if } F_{j} \leq F_{j}^{\min } \\ \frac{F_{j}^{\max }-F_{j}}{F_{j}^{\max }-F_{j}^{\min }} & \text { if } F_{j}^{\min } \leq F_{j} \leq F_{j}^{\max } \\ 0 & \text { if } F_{j} \geq F_{j}^{\max }\end{cases}$ [29]:

The standardized MF $\mu_{D}^{k}$ for each POF is determined as

$\mu_{D}^{k}=\frac{\sum_{i=1}^{2} \mu\left(F_{i}^{k}\right)}{\sum_{k=1}^{M} \sum_{i=1}^{2} \mu\left(F_{i}^{k}\right)}$

The solution which acquires a maximum $\mu_{D}^{k}$ value is the BCS of the MAMFED problem.

$$
\begin{aligned}
& \text { While } \sum_{i=1}^{n_{g}} \sum_{j=1}^{M_{i}} P_{i j}-\left(P_{D i}+P_{L i}+\sum_{z, z \neq i} T_{i z}\right)=0 \\
& \text { Randomly select a generator } i \text { of an individual } \\
& \text { Find } \Delta P_{i j}=\left|\sum_{i=1}^{n g} \sum_{j=1}^{M_{i}} P_{i j}-\left(P_{D i}+P_{L i}+\sum_{Z, Z \neq i} T_{i z}\right)\right| \\
& \text { If } \sum_{i=1}^{n_{g}} \sum_{j=1}^{M_{i}} P_{i j}-\left(P_{D i}+P_{L i}+\sum_{z, Z \neq i} T_{i z}\right) \neq 0 \\
& \text { Update } P_{i j}=\min \left(P_{i j}+\Delta P_{i j}, P_{i j, \max }\right) \\
& \text { Else } \\
& \text { Update } P_{i j}=\max \left(P_{i j}-\Delta P_{i j}, P_{i j, \text { min }}\right) \\
& \text { End if }
\end{aligned}
$$

Fig. 2 Equality constraint handling mechanism 
$\operatorname{Max}\left\{\mu_{D}^{k}: k=1,2, \ldots, M\right\}$

Fig. 3 Flow chart of FCFLBO algorithm for solving the MAMFED problem

\section{Implementation of FCFLBO algorithm for MAMFED problem}

The main steps involved in the FCFLBO algorithm for solving the MAMFED problem are as follows.

Step 1: initial feasible solutions are randomly generated. The fuel cost and emission objectives are

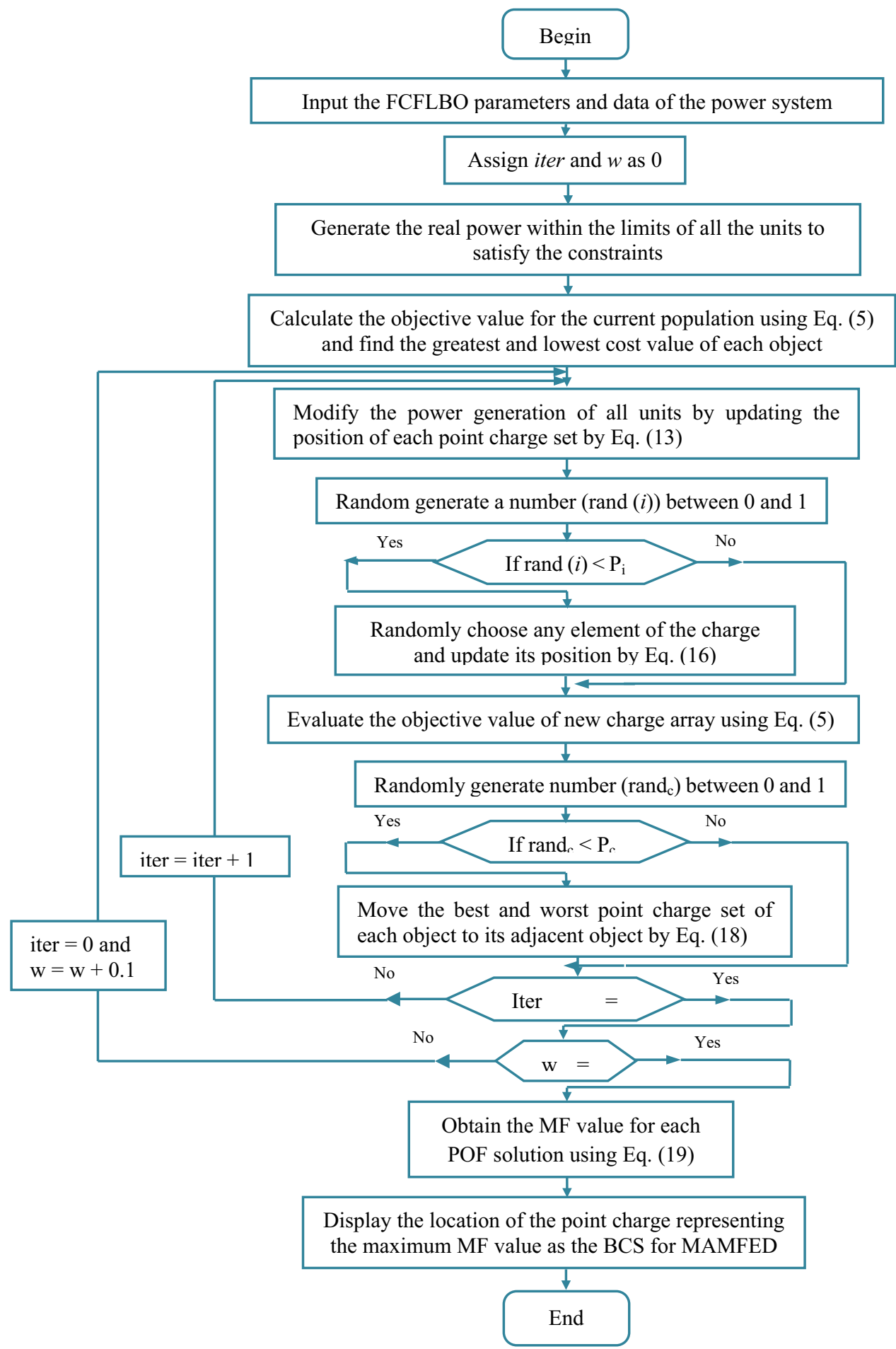


transferred into single objective function by Eq. 5 and their values are determined.

Step 2: The position of the generation of each unit is varied by implementing fascination/repugnance, probabilistic ionization and contact stages using Eqs. 15, 16, 17, 18, 19 and 20.

Step 3: The power generation and tie-line constraints are satisfied by Eqs. 12 and 13 respectively. For satisfying the power balance constraints (Eq. 11), an equality constraint handling strategy which is described in Fig. 2 is applied.

Step 4: The objective values of all the point charges are evaluated. Steps 2 to 3 are repeated until the stopping criterion is met.

Step 5: Finally, the fuzzy decision strategy is employed to acquire the BCS from the POF set.

The detailed step-by step procedure of FCFLBO based MAMFED problem is explained in Fig. 3.

\section{Numerical results and discussion}

To demonstrate the effectiveness of the suggested FCFLBO approach, it is applied to a three-area, 10-unit power system for the SAMFED and MAMFED problems. The power demand of the test system is chosen as $2700 \mathrm{MW}$. The coefficients of multi-fuel cost and emissions, generation limits of generating unit, and tie-line capacity limits are given in "Appendix". The suggested FCFLBO approach is executed in Matlab 7.1 and executed on an Intel core i3 processor with 4 GB RAM PC. In order to compare the performance of the FCFLBO algorithm, the SAMFED and MAMFED problems are also solved using KHA and EMA approaches. The FCFLBO, KHA and EMA approaches are executed for 50 autonomous trials to assess the solution quality and convergence behavior. The accompanying four scenarios are researched and the results of the suggested FCFLBO approach are compared with KHA, EMA and other state-of-art heuristic approaches surfaced in the literature.

Table 1 Optimal FCFLBO parameters

\begin{tabular}{ll}
\hline Parameter & Value \\
\hline Maximum number of objects $(n)$ & 5 \\
Population size of each object $(q)$ & 20 \\
Maximum number of iterations $\left(\right.$ iter $\left._{\text {max }}\right)$ & 200 \\
lonization probabilistic constant $\left(p_{i}\right)$ & 0.1 \\
Contact phase probabilistic constant $\left(P_{c}\right)$ & 0.5 \\
\hline
\end{tabular}

Scenerio 1: Minimization of the total fuel cost in the SAMFED problem,

Scenerio 2: Minimization of the total pollutant emissions in the SAMFED problem,

Scenerio 3: Minimization of both the total fuel cost and the total emission level simultaneously in the SAMFED problem, and

Scenerio 4: Minimization of the total fuel cost in the MAMFED problem

\subsection{Parameter selection}

In the FCFLBO approach, 5 main parameters that have to be predetermined are the number of objects, population size of each object, maximum number of iterations, probabilistic ionization, and contact constants. These parameters can be easily fixed depending on the complexity and scale of the considered SAMFED and MAMFED problems. The population size and maximum number of generations are fixed by trial and error method. The optimization procedure is terminated when there is no change in the result for 50 consecutive iterations or when 200 iterations are reached, whichever occurs first. The parameters selected for the suggested FCFLBO approach are given in Table 1. The accuracy or tolerance limit of the computation conducted using

Table 2 Optimal solution acquired by the suggested approach for scenario 1

\begin{tabular}{lll}
\hline Unit & Fuel Types & FCFLBO \\
\hline 1 & 2 & 218.2496 \\
2 & 1 & 211.6654 \\
3 & 1 & 280.9792 \\
4 & 3 & 239.8704 \\
5 & 1 & 278.7599 \\
6 & 3 & 239.3943 \\
7 & 1 & 288.5493 \\
8 & 3 & 239.3835 \\
9 & 3 & 428.2963 \\
10 & 1 & 274.4732 \\
Minimum cost $(\$ / \mathrm{h})$ & & 623.8613 \\
Emission $(\mathrm{kg} / \mathrm{h})$ & & 6460.9222 \\
\hline
\end{tabular}

Table 3 Performance comparison of the FCFLBO algorithm with other techniques for scenario 1

\begin{tabular}{ll}
\hline Approach & Fuel cost $(\$ / \mathrm{h})$ \\
\hline BSA & 623.9016 \\
KHA & 623.9545 \\
EMA & 623.9389 \\
FCFLBO & 623.8613 \\
\hline
\end{tabular}


Table 4 Optimal solution acquired by the FCFLBO algorithm for scenario 2

\begin{tabular}{lll}
\hline Unit & Fuel Types & FCFLBO \\
\hline 1 & 2 & 199.7561 \\
2 & 1 & 212.9532 \\
3 & 1 & 298.5494 \\
4 & 3 & 255.6735 \\
5 & 1 & 287.3095 \\
6 & 1 & 169.7462 \\
7 & 2 & 366.8659 \\
8 & 3 & 256.3758 \\
9 & 3 & 440 \\
10 & 1 & 212.7704 \\
Fuel cost (\$/h) & & 668.6195 \\
Minimum Emission $(\mathrm{kg} / \mathrm{h})$ & & 6044.4789 \\
\hline
\end{tabular}

Table 5 Performance comparison of the FCFLBO algorithm with other techniques for scenario 2

\begin{tabular}{ll}
\hline Approach & Emission $(\mathrm{kg} / \mathrm{h})$ \\
\hline KHA & 6051.8174 \\
EMA & 6054.5773 \\
FCFLBO & 6044.4789 \\
\hline
\end{tabular}

Table 6 Non-dominated solutions for various weighting values acquired by the suggested FCFLBO approach

\begin{tabular}{lllll}
\hline$W_{1}$ & $W_{2}$ & Fuel cost $(\$ / \mathrm{h})$ & Emission $(\mathrm{kg} / \mathrm{h})$ & $\begin{array}{l}\text { Membership } \\
\text { value }\left(\mu_{D}\right)\end{array}$ \\
\hline 1 & 0 & 623.8613 & 6460.9222 & 0.077760 \\
0.9 & 0.1 & 627.1618 & 6394.3657 & 0.085477 \\
0.8 & 0.2 & 630.0796 & 6327.4648 & 0.092783 \\
0.7 & 0.3 & 633.9257 & 6251.7609 & 0.100097 \\
$\mathbf{0 . 6}$ & $\mathbf{0 . 4}$ & $\mathbf{6 3 7 . 4 3 8 5}$ & $\mathbf{6 1 9 8 . 1 5 7 2}$ & $\mathbf{0 . 1 0 3 8 9 1}$ \\
0.5 & 0.5 & 642.4138 & 6161.9721 & 0.101887 \\
0.4 & 0.6 & 647.8515 & 6140.8452 & 0.096277 \\
0.3 & 0.7 & 652.3607 & 6117.9579 & 0.092621 \\
0.2 & 0.8 & 657.7984 & 6088.0282 & 0.088645 \\
0.1 & 0.9 & 663.3687 & 6066.9014 & 0.082802 \\
0 & 1 & 668.6193 & 6044.4789 & 0.077760 \\
\hline
\end{tabular}

Bold instances indicate the BCS from a POF set

Eqs. 6 and 10 for obtaining the optimal solutions is 0.0001 .

\subsection{Scenario 1}

Table 2 presents the optimal generation schedule acquired by the fuel cost minimization of the SAMFED using the suggested FCFLBO approach. In this scenario, the suggested approach is compared with the known approaches
Table 7 BCS acquired by the FCFLBO approach for scenario 3

\begin{tabular}{llc}
\hline Unit & Fuel Types & FCFLBO \\
\hline 1 & 2 & 209.3864 \\
2 & 1 & 212.9548 \\
3 & 1 & 294.2932 \\
4 & 3 & 252.7033 \\
5 & 1 & 285.8751 \\
6 & 1 & 195.8957 \\
7 & 2 & 300.7344 \\
8 & 3 & 245.6157 \\
9 & 3 & 425.6682 \\
10 & 1 & 276.8732 \\
Fuel cost $(\$ / \mathrm{h})$ & & 637.4385 \\
Emission $(\mathrm{kg} / \mathrm{h})$ & & 6198.1572 \\
\hline
\end{tabular}

such as EMA, KHA, and BSA [19] in Table 3. As can be seen from the results presented in Table 3, the FCFLBO approach outflanks the aforementioned approaches as far as the total fuel cost.

\subsection{Scenario 2}

The optimal values of generating units obtained by the FCFLBO for emission minimization are given in Table 4. The minimum emission value incurred from the FCFLBO algorithm is $6044.4789 \mathrm{~kg} / \mathrm{h}$. In order to demonstrate the effectiveness of the FCFLBO algorithm, the same problem is also solved using the KHA and EMA approaches. In Table 5, the pollutant emissions obtained from the suggested FCFLBO algorithm are compared with the KHA and EMA approaches. The results of this comparison demonstrate that the suggested approach is better than those of the KHA and EMA approaches. The FCFLBO algorithm obtains lesser emission level of $7.34 \mathrm{~kg} / \mathrm{h}$ and $10.1 \mathrm{~kg} / \mathrm{h}$ than those obtained using the KHA and EMA respectively.

\subsection{Scenario 3}

For this scenario, the total fuel cost and emission objectives of the SAMFED are simultaneously minimized. The objective function of the EED which is given in Eq. 5 is minimized. In order to provide the best trade-off, the weights $\mathrm{w}_{1}$ and $\mathrm{w}_{2}$ are varied in the range 0 to 1 in step of 0.1 so that their sum is one. The non-dominated solutions set acquired by the FCFLBO approach are depicted in Table 6 . The solution corresponding to the maximum membership value estimation is chosen as the BCS. It may be seen that the BCS are determined at $w_{1}=0.6$ and $w_{2}=0.4$ corresponding to $\mu_{D}=0.103891$. For the FCFLBO algorithm, the total fuel cost is $637.4385 \mathrm{\$} / \mathrm{h}$ and the total pollutant emissions of $6198.1572 \mathrm{~kg} / \mathrm{h}$. Table 7 presents the optimal 


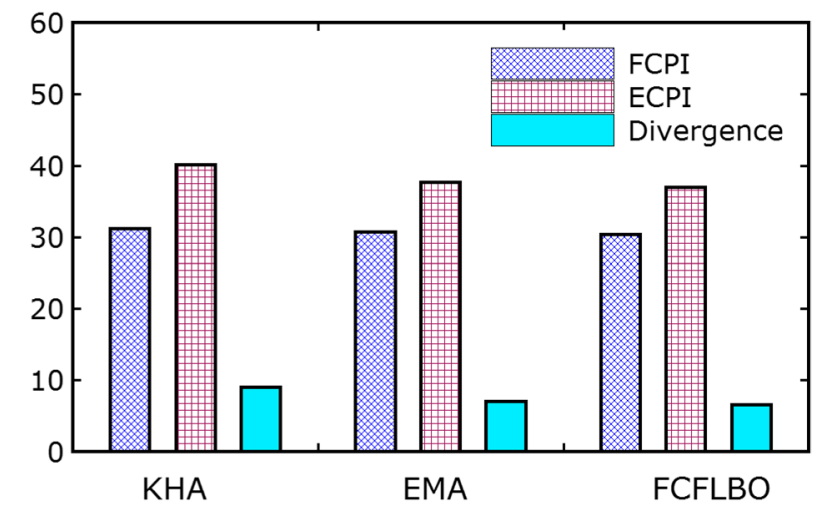

Fig. 4 Performance comparison of the FCFLBO algorithm with other techniques for scenario 3

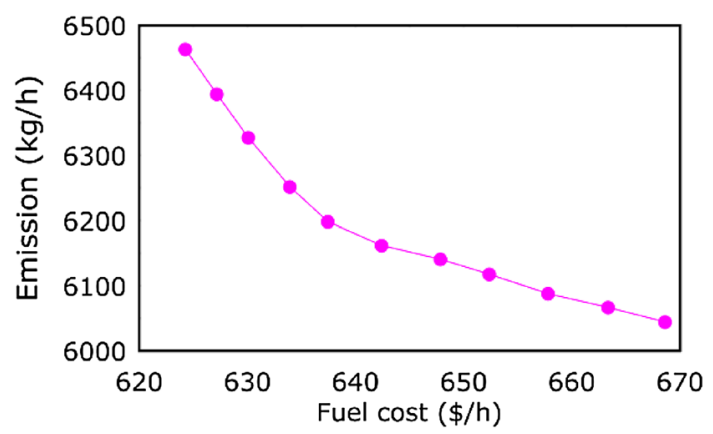

Fig. 5 POF curve of the proposed FCFLBO algorithm scenario 3

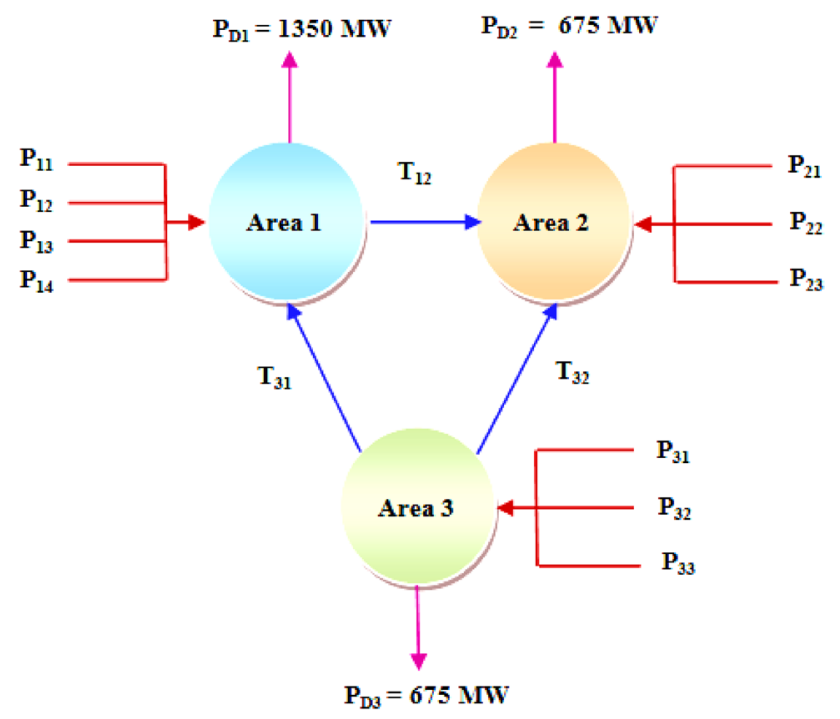

Fig. 6 Schematic diagram of three-area system

generation schedule of the SAMFED problem by minimizing both the total fuel cost and emissions.
Table 8 Best dispatch solution incurred by the suggested FCFLBO algorithm for scenario 4

\begin{tabular}{lllll}
\hline Unit & $\mathrm{P}_{\mathrm{ij}, \min }(\mathrm{MW})$ & $\mathrm{P}_{\mathrm{ij}, \max }(\mathrm{MW})$ & Fuel types & $\begin{array}{l}\text { Power gen- } \\
\text { eration (MW) }\end{array}$ \\
\hline $\mathrm{P}_{1,1}$ & 196 & 250 & 2 & 224.4524 \\
$\mathrm{P}_{1,2}$ & 157 & 230 & 1 & 212.1998 \\
$\mathrm{P}_{1,3}$ & 388 & 500 & 2 & 490.1287 \\
$\mathrm{P}_{1,4}$ & 200 & 265 & 3 & 240.5451 \\
$\mathrm{P}_{2,1}$ & 190 & 338 & 1 & 252.9731 \\
$\mathrm{P}_{2,2}$ & 200 & 265 & 3 & 234.5827 \\
$\mathrm{P}_{2,3}$ & 200 & 331 & 1 & 265.6763 \\
$\mathrm{P}_{3,1}$ & 200 & 265 & 3 & 234.9516 \\
$\mathrm{P}_{3,2}$ & 213 & 370 & 1 & 329.1937 \\
$\mathrm{P}_{3,3}$ & 200 & 362 & 1 & 251.0647 \\
$\mathrm{~T}_{21}$ & 100 & $\mathrm{P}_{\mathrm{L} 1}$ & 17.2541 & \\
$\mathrm{~T}_{31}$ & 99.9281 & $\mathrm{P}_{\mathrm{L} 2}$ & 9.8343 & \\
$\mathrm{~T}_{32}$ & 31.6022 & $\mathrm{P}_{\mathrm{L} 3}$ & 8.6804 & \\
Fuel cost (\$/h) & & & 653.8601 & \\
\hline
\end{tabular}

Table 9 Comparison of generation costs incurred by various approaches for scenario 4

\begin{tabular}{ll}
\hline Approach & Fuel cost $(\$ / \mathrm{h})$ \\
\hline RCGA [13] & 657.3 \\
EP [13] & 655.2 \\
DE [13] & 654 \\
ABC [13] & 654 \\
TLBO [14] & 653.99 \\
KHA & 654.72 \\
EMA & 654.6 \\
FCFLBO & 653.86 \\
\hline
\end{tabular}

The performance indices of EED problem such as fuel cost performance index (FCPI) and emission cost performance index (ECPI) are additionally performed. The FCPI and ECPI can be expressed as follows [28]:

$F C P I=\frac{F_{b c s}-F_{\min }}{F_{\max }-F_{\min }} \times 100$

$E C P I=\frac{E_{b c s}-E_{\min }}{E_{\max }-E_{\min }} \times 100$

The performance indices of the FCFLBO algorithm are compared with the results obtained using the KHA and EMA approaches, as shown in Fig. 4. It is evident that the performance indices acquired using the FCFLBO approach is superior to the aforementioned approaches as far as divergence. Figure 5 shows the Pareto optimal fronts (POF) curve of the suggested FCFLBO approach. It is seen that the POF curve of the suggested FCFLBO algorithm remains nearer to the axes, which implies that the FCFLBO approach offers better results. 
Fig. 7 Boxplots of multi-objective performance measures

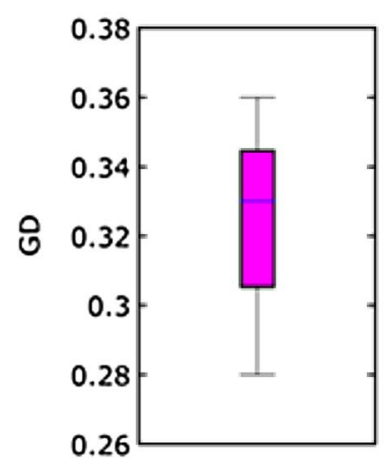

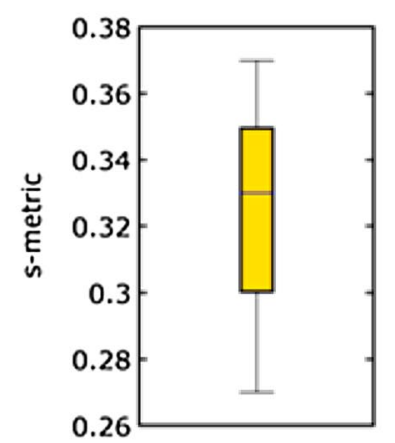

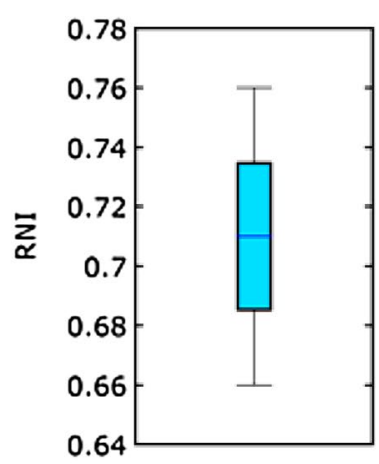

\subsection{Scenario 4}

In this scenario, the fuel cost minimization of MAMFED is addressed. Three areas, a 10-unit test system with transmission losses, and VPL and MFA impacts are taken into consideration. Areas 1, 2, and 3 comprise four, three and three generating units, respectively as displayed in Fig. 6 . The power demand of this system is $2700 \mathrm{MW}$. The power demand shares of areas 1, 2, and 3 are 50,25 , and $25 \%$ of total load demand respectively. The power stream from one area to another is restricted to $100 \mathrm{MW}$. Table 8 presents the simulation results obtained by the proposed FCFLBO approach. It can be easily seen that the optimal generation cost obtained by the FCFLBO approach is $653.8601 \$ / \mathrm{h}$, which is the lowest among the compared approaches. Area 1 imports power from areas 2 and 3 , and area 3 exports power to area 2. The comparison of the results of FCFLBO approach with those of EMA, KHA, RCGA [13], EP [13], DE [13], TLBO [14] and ABC [13] approaches is tabulated in Table 9 . The results show that the proposed strategy outperforms the other strategies considered with regard to finding the best generation schedule.

\subsection{Statistical analysis of the FCFLBO algorithm}

\subsubsection{Multi-objective performance indicators}

The multi-objective performance indicators such as, generational distance (GD), spacing metric (SM) and ratio of non-dominated individuals (RNI) are used to examine the performance of the suggested approach for Scenario 3. The smaller estimations of GD and SM demonstrate that better union to the POF curve, and better conveyance and assorted variety of the non-dominated solutions respectively. A closer estimation of one for RNI index demonstrates that the greatest number arrangements in a populace are non-dominated. The GD, SM, and RNI measures obtained by the proposed FCFLBO approach in 50 autonomous trials are shown as box and whisker plots

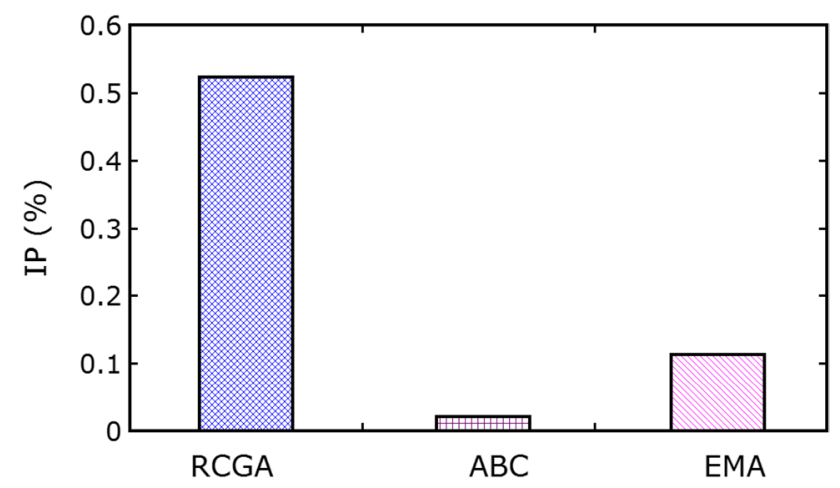

Fig. 8 Comparison of IP obtained by various approaches for scenario 4

Table 10 Comparison of function evaluation adopted by various scenarios

\begin{tabular}{lllll}
\hline Approach & Scenario 1 & Scenario 2 & Scenario 3 & Scenario 4 \\
\hline KHA & 268 & 272 & 436 & 452 \\
EMA & 259 & 258 & 424 & 443 \\
FCFLBO & 250 & 246 & 397 & 423 \\
\hline
\end{tabular}

Table 11 Comparison of CPU time adopted by various scenarios

\begin{tabular}{lllll}
\hline Approach & \multicolumn{4}{l}{ Average CPU time (s) } \\
\cline { 2 - 5 } & Scenario 1 & Scenario 2 & Scenario 3 & Scenario 4 \\
\hline KHA & 7.99 & 8.31 & 12.97 & 13.71 \\
EMA & 7.32 & 7.35 & 11.48 & 12.52 \\
FCFLBO & 6.08 & 6.13 & 9.72 & 10.23 \\
\hline
\end{tabular}

in Fig. 7. It tends to be seen from them that the FCFLBO algorithm has estimated the smaller values regarding $G D$ and SM indices, and more like one as for RNI indicator. 


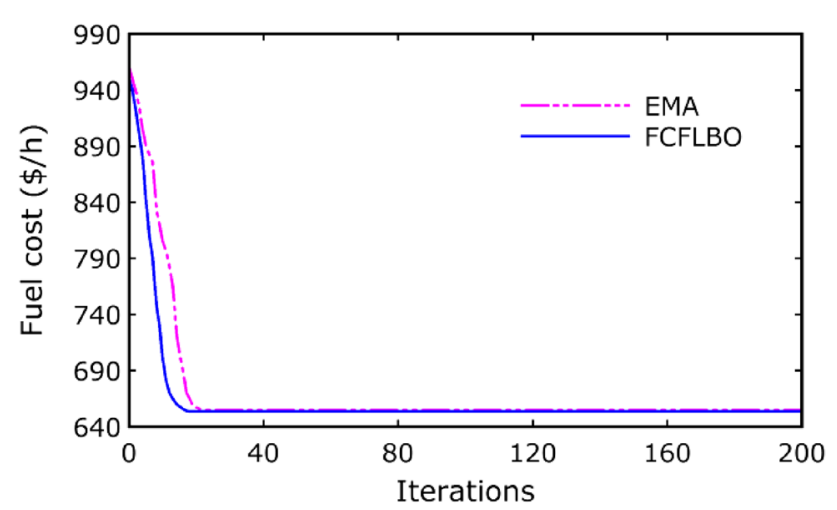

Fig. 9 Convergence comparison of the FCFLBO and EMA approaches

\subsubsection{Fuel cost improvement percentage}

Fuel cost improvement percentage (IP) is the ratio of fuel cost difference obtained between two approaches to the higher value of fuel cost obtained, expressed as a percentage, and is defined as follows:

$I P=\frac{\text { fuel cos } t \text { of the compared approach }- \text { fuel cos } t \text { of the suggested approach }}{\text { fuel cost of the compared approach }} \times 100$

The IPs obtained by the FCFLBO approach in comparison with the existing heuristic approaches for the case studies are shown in Fig. 8. The fuel cost IP of the FCFLBO approach compared to other approaches ranges from 0.113 to $0.5234 \%$ for scenario 4 . It is noteworthy that the IP of the FCFLBO approach is high for MAMFED with VPL impacts. Thus, the suggested FCFLBO approach provides better results than the other compared approaches.

\subsubsection{Computational Efficiency}

Tables 3, 4, 7, and 8 show that the minimum fuel costs achieved by the FCFLBO approach are $623.8613,668.6195$, 637.4385 , and $653.8601 \$ / \mathrm{h}$ for scenarios 1, 2, 3 and 4 respectively. These costs are lower than the ones presented in recent literature. The FCFLBO approach is, likewise, definitely effective. Tables 10 and 11 bestow the number of function evaluation and average CPU time adopted by the FCFLBO and EMA strategies for the various scenarios. It is significant that the number of function evaluation and CPU time are lesser than those of other referenced techniques. So, in general, it tends to be noted that the FCFLBO technique is more computationally effective than the EMA strategy.

\subsubsection{Convergence graph}

The convergence comparison of the EMA and FCFLBO approaches is shown in Fig. 9. It can well be construed that the FCFLBO approach requires a smaller number of iterations to converge to the globally optimal solution.

\section{Conclusion}

The MAMFED problem as an imperative issue in the modern power system analysis is solved in this paper. The optimal generation schedule of all committed units and power transfer between the areas through tie-lines are determined. The FCFLBO algorithm is used to solve the SAMFED and the MAMFED problems. The transmission line losses, VPL impacts, and tie-line limits of the power system are addressed. The bi-objective function is transferred into single objective function by weighted sum approach. Moreover, a fuzzy decision strategy is introduced to find one of the Pareto optimal fronts as the best comprised solution. In order to demonstrate the effectiveness of the FCFLBO algorithm in terms of solution quality, computational efficiency and convergence speed, it is solved to the MFED problems with three different scenarios and compared with the other state-of-the- approaches. In this research article, the merits are summarized hereunder.

- To the best of authors' knowledge, this research article is the first research work of applying FCFLBO approach for solving the SAMFED and the MAMFED problems.

- The FCFLBO algorithm validates the superior performance to provide the best POF for economic and emission dispatch problems with MFO.

- The difference between FCPI and ECPI obtained from FCFLBO is lesser than those obtained from the other compared approaches, indicating the superiority of FCFLBO in obtaining the BCS. Thus, it can be concluded that the FCFLBO algorithm is an efficient method of solving the MFMAED problem.

This research work not only offers an optimizer that provides an amazing advance for the MFMAED problem but also additionally advances the use of the evolutionary approaches in the energy optimization domain. For future work, it will be intriguing to implement this compelling approach to solve other economic operation problems of hybrid thermal wind-solar thermal power systems. 


\section{Compliance with ethical standards}

Conflict of interest On behalf of coauthor, the corresponding author states that there is no conflict of interest.

Open Access This article is licensed under a Creative Commons Attribution 4.0 International License, which permits use, sharing, adaptation, distribution and reproduction in any medium or format, as long as you give appropriate credit to the original author(s) and the source, provide a link to the Creative Commons licence, and indicate if changes were made. The images or other third party material in this article are included in the article's Creative Commons licence, unless indicated otherwise in a credit line to the material. If material is not included in the article's Creative Commons licence and your intended use is not permitted by statutory regulation or exceeds the permitted use, you will need to obtain permission directly from the copyright holder. To view a copy of this licence, visit http://creativecommons .org/licenses/by/4.0/.

\section{References}

1. Shoults RR, Chang SK, Helmick S, Grady WM (1980) A practical approach to unit commitment, economic dispatch and savings allocation for multiple-area pool operation with import/export constraints. IEEE Trans Power Apparatus Syst PAS-99:625-635

2. Quintana VH, Lopez R, Romano R, Valadez V (1981) Constrained economic dispatch of multi-area systems using the dantzigwolfe decomposition principle. IEEE Trans Power Apparatus Syst PAS-100:2127-2137

3. Ouyang Z, Shahidehpour SM (1991) Heuristic multi-area unit commitment with economic dispatch. IEE Proc C-Generation, Trans Distrib. https://doi.org/10.1049/ip-c.1991.0030

4. Wang C, Shahidehpour SM (1992) A decomposition approach to nonlinear multi-area generation scheduling with tie-line constraints using expert systems. IEEE Trans Power Syst 7:14091418. https://doi.org/10.1109/59.207362

5. Streiffert D (1995) Multi-area economic dispatch with tie line constraints. IEEE Trans Power Syst 10:1946-1951. https://doi. org/10.1109/59.476062

6. Yalcinoz T, Short MJ (1988) Neural networks approach for solving economic dispatch problem with transmission capacity constraints. IEEE Trans Power Syst 13:307-313. https://doi. org/10.1109/59.667341

7. Thang NT (2013) Economic emission load dispatch with multiple fuel options using hopfield lagrange network. Int J Adv Sci Technol 57:9-24

8. Li YZ, Jiang L, Wu QH, Wang P, Gooi HB (2017) Wind-thermal power system dispatch using MLSAD model and GSOICLW algorithm. Knowl Based Syst 116:94-101. https://doi.org/10.1016/j. knosys.2016.10.028

9. Jayabarathi T, Sadasivam G, Ramachandran V (2000) Evolutionary programming based multi-area economic dispatch with tie line constraints. Electr Machines Power Syst 28:1165-1176. https ://doi.org/10.1080/073135600449044

10. Manoharan PS, Kannan PS, Baskar S, Iruthayarajan M (2009) Evolutionary algorithm solution and KKT based optimality verification to multi-area economic dispatch. Int J Electr Power Energy Syst 31:365-373. https://doi.org/10.1016/j.ijepes.2009.03.010

11. Sharma M, Manjaree P, Laxmi S (2011) Reserve constrained multi-area economic dispatch employing differential evolution with time varying mutation. Int J Electr Power Energy Syst 33:753-766. https://doi.org/10.1016/j.ijepes.2010.12.033
12. Somasundaram P, Jothi Swaroopan NM (2011) Fuzzified particle swarm optimization algorithm for multi-area security constrained economic dispatch. Electr Power Components Syst 39:979-990

13. Basu M (2013) Artificial bee colony optimization for multi-area economic dispatch. Int J Electr Power Energy Syst 49:181-187. https://doi.org/10.1016/j.ijepes.2013.01.004

14. Basu M (2014) Teaching-learning-based optimization algorithm for multi-area economic dispatch. Energy 68:21-28. https://doi. org/10.1016/j.energy.2014.02.064

15. Basu M (2017) Fast convergence evolutionary programming for multi-area economic dispatch. Electr Power Components Syst 45:1629-1637. https://doi.org/10.1080/15325008.2017.1376234

16. Nguyen KP, Dinh ND, Fujita G (2015) Multi-area economic dispatch using hybrid cuckoo search algorithm. Proc 50th International Universities Power Engineering Conference, stoke on Trent, UK, pp 1-4. https://doi.org/10.1109/UPEC.2015.7339777

17. Ghasemi M, Aghaei J, Akbari E, Ghavidel S, Li L (2016) A differential evolution particle swarm optimizer for various types of multi-area economic dispatch problems. Energy 107:182-195. https://doi.org/10.1016/j.energy.2016.04.002

18. Zhang P, Ma W, Dong Y (2019) Multi-area economic dispatching using improved grasshopper optimization algorithm. Evol Syst. https://doi.org/10.1007/s12530-019-09320-6

19. Modiri-Delshad M, Aghay Kaboli SH, Taslimi-Renani E (2016) Backtracking search algorithm for solving economic dispatch problems with valve-point effects and multiple fuel options. Energy 116:637-649. https://doi.org/10.1016/j.energ y.2016.09.140

20. Ghorbani, Babaei E, Sadikoglu F (2016) Exchange market algorithm for economic load dispatch. Int J Electr Power Energy Syst 75:19-27

21. Mandal B, Roy PK, Mandal S (2014) Economic load dispatch using krill herd algorithm. Int J Electr Power Energy Syst 57:1-10

22. Mokarram MJ, Niknam T, Aghaei J, Shafie-khah M, Catalao JPS (2019) Hybrid optimization algorithm to solve the nonconvex multiarea economic dispatch problem. IEEE Syst J 13:34003409. https://doi.org/10.1109/JSYST.2018.2889988

23. Mokarram MJ, Gitizadeh M, Niknam T, Niknam S (2019) Robust and effective parallel process to coordinate multi-area economic dispatch (MAED) problems in the presence of uncertainty. IET Gen Transm Distrib 13:4197-4205. https://doi.org/10.1049/ iet-gtd.2019.0319

24. Qin J, Wan Y, Yu X, Kang Y (2020) A newton method-based distributed algorithm for multi-area economic dispatch. IEEE Trans Power Syst 35:986-996. https://doi.org/10.1109/TPWRS .2019.2943344

25. Zare M, Narimani MR, Malekpour M, Azizipanah-Abarghooee $\mathrm{R}$, Terzija V (2021) Reserve constrained dynamic economic dispatch in multi-area power systems: an improved fireworks algorithm. Int J Elect Power Energy Syst 126A:e106579. https://doi. org/10.1016/j.ijepes.2020.106579

26. Chaudhary V, Dubey HM, Pandit M, Bansal JC (2020) Multi-area economic dispatch with stochastic wind power using salp swarm algorithm. Array 8:e00044. https://doi.org/10.1016/j. array.2020.100044

27. Sakthivel VP, Suman M, Sathya PD (2020) Squirrel search algorithm for economic dispatch with valve-point effects and multiple fuels. Energy Sources Part B 15:351-382. https://doi. org/10.1080/15567249.2020.1803451

28. Sakthivel VP, Suman M, Sathya PD (2020) Nonconvex economic environmental load dispatch using fuzzy based squirrel search algorithm. Int J Energy Convers 8:61-70 
29. Sakthivel VP, Suman M, Sathya PD (2020) Environmental/economic dispatch problem: Coulomb's and Franklin's laws based optimization algorithm. Int Rev Electr Eng 15:421-430

30. Ghasemi M, Ghavidel S, Aghaei J, Akbari E, Li L (2018) CFA optimizer: a new and powerful algorithm inspired by franklin's and coulomb's laws theory for solving the economic load dispatch problems. Int Trans Electr Energy Syst 28:e2536. https://doi. org/10.1002/etep.2536

Publisher's Note Springer Nature remains neutral with regard to jurisdictional claims in published maps and institutional affiliations. 